\title{
Reviewers: World Trade Review 2018
}

The World Trade Review depends on its reviewers to maintain and improve the quality of the articles that it publishes. The Editorial Board is very grateful to the following, who have acted as reviewers, sometimes more than once.

Dukgeun Ahn, Seoul National University, Korea.

Per Altenberg, Kommerskollegium, National Board of Trade, Sweden.

Mohsen Bahmani-Oskooee, University of Wisconsin, USA.

Lorand Bartels, University of Cambridge, UK.

Axel Berger, Deutsches Institut fůr Entwicklungspolitik, Germany.

Chad Bown, Peterson Institute for International Economics, UK.

Tomer Broude, Hebrew University of Jerusalem, Israel.

Mira Burri, Universität Luzern, Switerland.

Liesbeth Casier, International Institute for Sustainable Development, Switzwerland.

Anupam Chander, University of California, Davis, USA.

Byunghee Chang, Sungkyun Kwan University, Korea.

Pao-Li Chang, Singapore Management University, Singapore.

Steve Charnovitz, The George Washington University Law School, USA

Adam Chilton, University of Chicago, USA.

Sungjoon Cho, Chicago-Kent College of Law, USA.

Kasturi Das, Institute of Management Technology, India.

Bill Davey, University of Illinois, USA.

Jaime de Melo, University of Geneva, Switzerland.

Charalampos Efstathopoulos, Aberystwyth University, UK.

Manfred Elsig, University of Bern, Switzerland.

Adrian Ely, University of Sussex, UK.

Ilaria Espa, World Trade Institute, Switzerland.

Rod Falvey, Bond University, Australia.

Ana Fernandes, The World Bank, USA.

Carsten Fink, World Intellectual Property Organisation, Switzerland.

James Flett, The European Commission, Belgium.

David Gantz, University of Arizona, USA.

David Gisselquist, Independent Scholar, USA.

Keith Head, University of British Columbia, Canada.

Cristina Herghelegiu, Université Paris, France.

Lisa Herzog, Technical University, Munich, Germany.

Jason Houston-McMillan, World Trade Organisation, Switzerland. 
Gary Hufbauer, Peterson Institute for International Economics, USA.

Rieko Ishii, Shiga University, Japan.

Merit Janow, Columbia University, USA.

Melise Jaud, University of Lausanne, Switzerland.

Robert Koopman, The World Trade Organisation, Switzerland.

Markus Krajewski, Friedrich-Alexander Universität, Erlangen-Nürnberg, Germany.

Zornitsa Kutlina-Dimitrova, The European Commission, Belgium.

James Lake, Southern Methodist University, Dallas, USA.

Arjan Lejour, CPB Bureau for Economic Policy Analysis, Netherlands.

Emily Lydgate, University of Sussex, UK.

Edward Mansfield, University of Pennsylvania, USA.

Gabrielle Marceau, The World Trade Organisation, Switzerland.

Keith Maskus, University of Colorado, Boulder, USA.

Mislav Mataija, The European Commission.

Helen Milner, Princeton University, USA.

Sebastien Miroudot, Organisation of Economic Cooperation and Development, France.

Amrita Narlikar, German Institute of Global and Area Studies, Germany.

Hildegunn Nordas, Organisation of Economic Cooperation and Development, France.

Allesandro Olper, Universita degli Studi di Milano, Italy.

Gianluca Orefice, CEPII, France.

Hezron Osano, Technical University of Kenya, Kenya.

Delimatsis Panagiotis, Tilburg University, Netherlands.

Shin-yi Peng, National Tsing Hua University, Taiwan.

Michael Plummer, Johns Hopkins University, USA.

Vitaliy Pogeretskyy, Advisory Centre on WTO Law, Switzerland.

Rodrigo Polanco, World Trade Institute, Switzerland.

Mando Rachovitsa, Rijksuniversiteit Gronignen, Netherlands.

Luca Rubini, University of Birmingham, UK.

André Sapir, Université de Bruxelles, Belgium.

Yulia Selivanova, International Centre for Trade and Sustainable Development, Switzerland.

Sherzod Shadikhodjaev, KDI School of Public Policy and Management, Korea.

Gergory Shaffer, University of California, Irvine, USA.

Anirudh Shingal, European University Institute, Italy.

Gabriele Spilker, Universität Salzburg. Austria.

Cristian Tietje, Universität Halle-Wittenberg, Germany.

Umut Turksen, Coventry University, UK.

Erik van der Marel, European Centre for International Political Economy, Belgium.

Mauro Vigani, University of Gloucestershire, UK.

Bruce Wardhaugh, University of Manchester, UK.

Rorden Wilkinson, University of Sussex, UK.

Jason Winfree, University of Idaho, USA. 
Robert Wolfe, Queen's University, Canada.

Mark Wu, Harvard University, USA.

Mauricio Zanardi, Lancaster University, UK.

Zhen Zhu, IMT Institute for Advanced Studies, Lucca, Italy. 